\title{
Harary Polynomials
}

Orli Herscovici*, Johann A. Makowsky ${ }^{\ddagger}$ and Vsevolod Rakita ${ }^{\dagger}$

\author{
* Department of Mathematics, University of Haifa, Israel \\ Email: orli.herscovici@gmail.com \\ $\ddagger$ Department of Computer Science, Technion-Israel Institute of Technology, Haifa, Israel \\ Email: janos@cs.technion.ac.il \\ ${ }^{\dagger}$ Department of Mathematics, Technion-Israel Institute of Technology, Haifa, Israel \\ Email:vsevolod@campus.technion.ac.il
}

Received: November 13, 2020, Accepted: February 7, 2021, Published: February 19, 2021 The authors: Released under the CC BY-ND license (International 4.0)

\begin{abstract}
Given a graph property $\mathcal{P}, \mathrm{F}$. Harary introduced in $1985 \mathcal{P}$-colorings, graph colorings where each color class induces a graph in $\mathcal{P}$. Let $\chi_{\mathcal{P}}(G ; k)$ counts the number of $\mathcal{P}$-colorings of $G$ with at most $k$ colors. It turns out that $\chi_{\mathcal{P}}(G ; k)$ is a polynomial in $\mathbb{Z}[k]$ for each graph $G$. Graph polynomials of this form are called Harary polynomials. In this paper we investigate properties of Harary polynomials and compare them with properties of the classical chromatic polynomial $\chi(G ; k)$. We show that the characteristic and the Laplacian polynomial, the matching, the independence and the domination polynomials are not Harary polynomials. We show that for various notions of sparse, non-trivial properties $\mathcal{P}$, the polynomial $\chi_{\mathcal{P}}(G ; k)$ is, in contrast to $\chi(G ; k)$, not a chromatic, and even not an edge elimination invariant. Finally, we study whether the Harary polynomials are definable in monadic second-order Logic.
\end{abstract}

Keywords: Generalized colorings; Graph polynomials; Courcelle's Theorem

2020 Mathematics Subject Classification: 05; 05C30; 05C31

\section{Introduction and main results}

\subsection{Prelude}

This paper initiates a systematic study of univariate graph polynomials, called Harary polynomials, or generalized chromatic polynomials. We explore how the Harary polynomials differ from the traditional univariate graph polynomials from the literature, among them the characteristic and Laplacian polynomial, the original chromatic polynomial, the matching polynomial, the independence and the clique polynomial.

The paper uses techniques developed in the last twenty years by the second author and his collaborators, I. Averbouch, B. Godlin, T. Kotek and E. Ravve, and shows that these techniques form a solid body of tools, which can be applied to study Harary polynomials. The results show a coherent picture, even if no new techniques are developed in this paper.

\subsection{Harary polynomials}

Let $\mathcal{P}$ be a graph property. In [19] F. Harary introduced the notion of $\mathcal{P}$-coloring as a generalization of proper colorings, which he called conditional colorings. Let $G=(V(G), E(G))$ be a graph and $[k]=\{1,2, \ldots, k\}$. A function $c: V(G) \rightarrow[k]$ is a $\mathcal{P}$-coloring with at most $k$ colors if for every $i \in[k]$ the set $\left\{v \in V(G): f^{-1}(i)\right\}$ induces a graph in $\mathcal{P}$. If $\mathcal{P}$ is the property that $E(G)=\emptyset$, i.e., $\mathcal{P}$ consists of all the edgeless graphs, this gives the proper colorings. Other properties of $\mathcal{P}$ studied in the literature are: $G$ is connected, $G$ is triangle-free or $G$ is a complete graph. F. Harary introduced $\mathcal{P}$-colorings with the idea that they might behave in a similar way to proper colorings. $\mathcal{P}$-colorings were further studied in [11].

Let $\chi_{\mathcal{P}}(G ; k)$ be the number of $\mathcal{P}$-colorings of $G$ with color set $[k]$, and $\chi_{\mathcal{P}}(G)$ be the $\mathcal{P}$-chromatic number, which is the least $k$ such that $G$ has a $\mathcal{P}$-coloring.

\footnotetext{
* Supported by the Israel Science Foundation (grant No. 1144/16)

† Supported by a grant of the Graduate School of the Technion-Israel Institute of Technology
} 
$\chi(G ; k)$ is the chromatic polynomial, i.e., the Harary polynomial for $\mathcal{P}$ containing all the edgeless graphs. Generalizing Birkhoff's Theorem from 1912 for $\chi(G ; k)$ it was noted in [33] that for every finite graph $G$ the counting function $\chi_{\mathcal{P}}(G ; k)$ is a polynomial in $\mathbb{Z}[k]$, see also [21]. The family of polynomials $\chi_{\mathcal{P}}(G ; k)$ indexed by graphs $G$ is a graph polynomial called a Harary polynomial in [31], which can be written as

$$
\chi_{\mathcal{P}}(G ; x)=\sum_{i \geq 1} b_{i}^{\mathcal{P}}(G) x_{(i)},
$$

where $b_{i}^{\mathcal{P}}(G)$ is the number of partitions of $V(G)$ into $i$ non-empty parts, where each part induces a graph in $\mathcal{P}$, and $x_{(i)}$ is the falling factorial.

Facts 1.1. For every graph property $\mathcal{P}$ and every graph $G$ of order $n$ we have

(i) $b_{0}^{\mathcal{P}}(G)=0$, if the empty graph $(\emptyset, \emptyset)$ is not in $\mathcal{P}$.

(ii) $b_{1}^{\mathcal{P}}(G) \in\{0,1\}$ and $b_{1}^{\mathcal{P}}(G)=1$ if and only if $G \in \mathcal{P}$.

(iii) $b_{n}^{\mathcal{P}}(G) \in\{0,1\}$ and $b_{n}^{\mathcal{P}}(G)=1$ if and only if $K_{1} \in \mathcal{P}$. In other words, the polynomial $\chi_{\mathcal{P}}(G ; k)$ is monic of degree $|V(G)|$ if and only if $K_{1} \in \mathcal{P}$.

(iv) If $k<n$ and $\chi_{\mathcal{P}}(G ; k)=0$ then for all $0<\ell<k$ also $\chi_{\mathcal{P}}(G, \ell)=0$.

Examples 1.1. Here are some $\mathcal{P}$-colorings and Harary polynomials studied in the literature.

(i) mcc $_{t}$-colorings. Here $\mathcal{P}=\mathcal{P}_{t}$, where $\mathcal{P}_{t}$ is the graph property such that the connected components of $H \in \mathcal{P}_{t}$ have order at most $t$.

For $t=1$ these are the proper colorings, for $t=2$ these are the $\mathcal{P}_{3}$-free colorings. They were introduced in [26] with a slightly different notation.

(ii) Let $H$ be a connected graph of order $t . D U(H)$ consisting of non-empty disjoint unions of copies of $H$. $D U(H)$-colorings are $m c c_{t}$-colorings. They are studied in [18].

(iii) Let $\operatorname{Fr}(H)$ be the class of all graphs which do not contain $H$ as an induced subgraph, which we call $H$-free graphs. $\operatorname{Fr}(H)$-colorings are studied in $[1,10,11] . K_{2}$-free colorings are just the proper colorings.

(iv) A graph property $\mathcal{P}$ is additive if it is closed under forming disjoint unions. $\mathcal{P}$ is hereditary if it is closed under induced subgraphs. A coloring is $\mathcal{A H}$ if it is a $\mathcal{P}$-coloring for some $\mathcal{P}$ which is both additive and hereditary. $\mathcal{A H}$-colorings were studied in [15].

(v) The adjoint polynomial $A(G ; x)=\chi_{\mathcal{P}}(G ; k)$ is defined by taking $\mathcal{P}$ to be the class of all complete graphs. It was introduced in [27], see also [8].

(vi) If $\mathcal{P}$ consists of all connected graphs, we speak of convex colorings, and put $C(G ; x)=\chi_{\mathcal{P}}(G ; k)$, see $[18,37,38]$.

The purpose of this paper is to initiate the study of Harary polynomials by comparing them to the chromatic polynomial.

\subsection{The chromatic polynomial and edge elimination invariants}

One of the fundamental properties of the chromatic polynomial is its characterization via edge elimination properties. Given a graph $G$ and an edge $e \in E(G)$ we denote by $G_{-e}, G_{/ e}$ and $G_{\dagger e}$ the graphs obtained from $G$ by deleting, contracting and extracting the edge $e$. Extraction deletes $e=(u, v)$ together with the vertices $u, v$ and all the edges incident with $u$ or $v$. A graph parameter $p(G)$ is an edge elimination (EE) invariant, see [6], if it can be written as a certain linear combination of $p\left(G_{-e}\right), p\left(G_{/ e}\right), p\left(G_{\dagger e}\right)$.

It is well known that $\chi(G ; k)$ is an EE-invariant even without using $p\left(G_{\dagger e}\right)$. Other EE-invariants are the matching polynomials, some version of the Tutte polynomial and many others, [40,41]. However, the original Tutte polynomial is not an EE-invariant. An alternative name for EE-invariants is DCE-invariants, for Deletion, Contraction and Extraction.

Theorem 1.1 (See $[5,6])$. There is a graph polynomial $\xi(G ; x, y, z)$

$$
\begin{aligned}
\xi(G ; x, y, z)= & \sum_{A, B \subseteq E(G)} x^{c(A \cup B)-\operatorname{cov}(B)} y^{|A|+|B|-\operatorname{cov}(B)} z^{\operatorname{cov}(B)}= \\
& \sum_{A, B \subseteq E(G)} x^{c(A \cup B)} y^{|A|+|B|}\left(\frac{z}{x y}\right)^{\operatorname{cov}(B)}
\end{aligned}
$$

such that 
(i) $\xi(G ; x, y, z)$ is an edge elimination invariant.

(ii) $\xi(G ; x, y, z)$ is universal, i.e., every other graph parameter $p(G)$ which is an edge elimination invariant is a substitution instance of $\xi(G ; x, y, z)$, i.e., it can be obtained from $\xi(G ; x, y, z)$ by substituting or replacing $x, y, z$ by a polynomial in the indeterminates $x, x^{-1}, y, y^{-1}, z, z^{-1}$.

Here

- the summation is over $A, B \subseteq E(G)$ such that the vertex subsets $V(A), V(B)$ covered by $A$ and $B$, respectively, are disjoint,

- $c(A)$ is the number of connected components in $(V(G), A)$, and

- $\operatorname{cov}(B)$ is the number of covered (connected) components of B, i.e. the number of connected components of $(V(B), B)$.

\subsection{MSOL-definable graph polynomials}

The language of graphs has one binary relation symbol for the edge relation. If we fix $k$ we note that $\chi(G ; k)>0$ if and only if $G$ is $k$-colorable. This can be expressed by a formula in monadic second-order logic MSOL in the language of graphs by the formula

$$
\exists V_{1} \exists V_{2} \ldots \exists V_{k}\left(\operatorname{Partition}\left(V_{1}, V_{2}, \ldots, V_{k}\right) \wedge \bigwedge_{i=1}^{k} \operatorname{Indep}\left(V_{i}\right)\right) .
$$

$\operatorname{Partition}\left(V_{1}, V_{2}, \ldots, V_{k}\right)$ and $\operatorname{Indep}\left(V_{i}\right)$ are first-order expressible in the language of graphs. The same works for Harary polynomials provided $\mathcal{P}$ is MSOL-definable. Checking whether a graph $G$ is $k$-colorable is NPcomplete. For the complexity of checking whether a graph is $\mathcal{P}$-colorable for various graph properties $\mathcal{P}$, the reader may consult $[1,10,18]$.

However, using Courcelle's celebrated Theorem, [14, Chapter 13] and [16, Chapter 11], MSOL-definability implies that checking whether a graph $G$ is $k$-colorable is fixed parameter tractable (FPT) for graphs of bounded tree-width, and even for graphs of bounded clique-width or rank-width.

For the chromatic polynomial one looks at the problem of computing the value of $\chi(G ; k)$ for given $k$ as a function of $G$. For $k=0,1,2$ this is computable in polynomial time, whereas for $k \geq 3$ this is $\sharp \mathbf{P}$-complete, [25]. For graphs of fixed tree-width $w$, this is still in FPT. To see this one can use an extension of Courcelle's Theorem to the class of MSOL-definable graph polynomials, [13].

The language of hypergraphs has two unary predicates $V$ and $E$ for vertices and edges which partition the universe, and a binary incidence relation $R$ saying that vertices are connected by edges. We denote by $\mathrm{MSOL}_{g}$ $\left(\mathrm{MSOL}_{h}\right)$ the monadic second-order logic in the language of graphs (hypergraphs).

Proposition 1.1. Let $\mathcal{P}$ be a graph property definable in $\mathrm{MSOL}_{g}\left(\mathrm{MSOL}_{h}\right)$. Then checking whether a graph $G$ is $\mathcal{P}$-colorable with $k$ colors is definable in $\mathrm{MSOL}_{g}\left(\mathrm{MSOL}_{h}\right)$.

Theorem 1.2 (See [30]). $\chi(G ; k)$ is not an $\mathrm{MSOL}_{g}$-definable polynomial, but it is $\mathrm{MSOL}_{h}$-definable.

Proof. For fixed $k$ we write

$$
\exists U_{1}, \ldots, \exists U_{k} \bigwedge_{j \in[k]} \phi_{\mathcal{P}}\left(U_{j}\right)
$$

where $U_{j}$ are sets of vertices and $\phi_{\mathcal{P}}\left(U_{j}\right)$ says that $U_{j}$ induces a graph in $\mathcal{P}$, provided $U_{j}$ is not empty.

Theorem 1.3. The most general EE-invariant $\xi(G ; x, y, z)$ is $\mathrm{MSOL}_{h}$-definable for graphs with a linear order on the vertices. Furthermore, this definition is invariant under the particular order of the vertices.

As there is no published proof of this, we include a proof here in the Appendix A. To prove that $\chi(G ; k)$ is not $\mathrm{MSOL}_{g}$-definable we use the method of connection matrices, explained in Section 5 . To prove that $\chi(G ; k)$ is $\mathrm{MSOL}_{h}$-definable we use that $\chi(G ; k)$ is an EE-invariant and Theorems 1.1 and 1.3. We do not know a direct method, without the use of an $\mathrm{MSOL}_{h}$-definable EE-invariant, to show that $\chi(G ; k)$ is indeed $\mathrm{MSOL}_{h}$-definable.

Theorem 1.2 still implies that evaluating $\chi(G ; k)$ is fixed parameter tractable (FPT) for graphs of tree-width at most $w$, whereas for graphs of clique-width $w$ this is still open, [7,32]. 


\subsection{Main results}

A graph property $\mathcal{P}$ is trivial if it is empty, finite (up to isomorphisms), or it contains all finite graphs. Our main question in this paper asks whether Courcelle's Theorem and its variations can be applied to Harary polynomials for non-trivial graph properties. This amounts to asking:

(i) Are there non-trivial graph properties $\mathcal{P}$ such that the Harary polynomial $\chi_{\mathcal{P}}(G, x)$ is $\mathrm{MSOL}_{g}$-definable?

(ii) Are there non-trivial graph properties $\mathcal{P}$ such that the Harary polynomial $\chi_{\mathcal{P}}(G, x)$ is an EE-invariant and hence $\mathrm{MSOL}_{h}$-definable?

Recall that a graph property $\mathcal{P}$ is hereditary (monotone, minor-closed) if it is closed under taking induced subgraphs (subgraphs, minors). Clearly, if $\mathcal{P}$ is minor-closed, it is also monotone, and if $\mathcal{P}$ is monotone, it is also hereditary.

A graph property $\mathcal{P}$ is ultimately clique-free if there exists $t \in \mathbb{N}$ such that no graph $G \in \mathcal{P}$ contains a $K_{t}$, i.e., a complete graph of order $t$. Analogously, $\mathcal{P}$ is ultimately biclique-free if there exists $t \in \mathbb{N}$ such that no graph $G \in \mathcal{P}$ has $K_{t, t}$ as a subgraph (not necessarily induced). $K_{t, t}$ is the complete bipartite graph of order $2 t$. Clearly, biclique-free implies clique-free, but not conversely.

Theorem 1.4. Let $\mathcal{P}$ be a graph property and $\chi_{\mathcal{P}}(G, x)$ the Harary polynomial associated with $\mathcal{P}$.

(i) If $\mathcal{P}$ is hereditary, monotone, or minor-closed, then $\chi_{\mathcal{P}}(G ; x)$ is an EE-invariant iff $\chi_{\mathcal{P}}(G ; x)$ is the chromatic polynomial $\chi(G ; x)$.

(ii) If $\mathcal{P}$ is ultimately clique-free (biclique-free), $\chi_{\mathcal{P}}(G ; x)$ is not $\mathrm{MSOL}_{g}$-definable.

The proof of (i) appears as Theorem 3.3, and the proof of (ii) appears as Theorem 5.2.

Remark 1.1. If $\mathcal{P}$ consists of all complete graphs or all connected graphs, $\mathcal{P}$ is not ultimately clique-free, hence Theorem 1.4 does not apply to the Harary polynomials $A(G ; x)$ and $C(G ; x)$. Nevertheless, analogue results are presented in Sections 3 and 5.

\subsection{Sparsity}

For a systematic study of sparsity (and density) of graph properties see [34,35].

Theorem 1.5. (i) Turan's Theorem ([42] and [17, Chapter 8.3]):

Let $G$ be $K_{t}$-free. Then $|E(G)| \leq\left(1-\frac{1}{r}\right) \frac{n^{2}}{2}$.

(ii) ( [23]) Let $G$ be $K_{t, t}$-free. Then $|E(G)|=O\left(n^{2-\frac{1}{t}}\right)$.

(iii) ([39]) If a graph property $\mathcal{P}$ is nowhere dense or degenerate (or equivalently uniformly sparse) then $\mathcal{P}$ is ultimately biclique-free.

(iv) ([39]) There are graph properties $\mathcal{P}_{1}, \mathcal{P}_{2}$ which are both ultimately biclique-free but $\mathcal{P}_{1}$ is not degenerate and $\mathcal{P}_{2}$ is not nowhere dense.

In the light of Theorem 1.5 ultimately biclique-free is renamed to weakly sparse in [36]. However, ultimately clique-free graphs can be rather dense, with $c(t) \cdot n^{2}$ edges rather than $n^{2-\epsilon(t)}$ edges.

Theorem 1.4 together with Theorem 1.5 shows that Harary polynomials which are EE-invariants or $\mathrm{MSOL}_{g^{-}}$ definable have to be defined using dense properties $\mathcal{P}$ as required by Turán's Theorem.

\section{Graph polynomials which are not Harary polynomials}

Many familiar graph polynomials are not Harary polynomials of the form $\chi_{\mathcal{P}}(G ; x)$. We generalize here $[31$, Theorem 5.7].

Lemma 2.1. For every graph property $\mathcal{P}$ we have

$$
\chi_{\mathcal{P}}(G ; 1)= \begin{cases}1 & G \in \mathcal{P}, \\ 0 & G \notin \mathcal{P} .\end{cases}
$$

Using Lemma 2.1 we get

Proposition 2.1. Let $F(G ; x)$ be a graph polynomial and $G$ be a graph such that $F(G ; 1) \neq 0$ and $F(G ; 1) \neq 1$. Then there is no graph property $\mathcal{P}$ such that $\chi_{\mathcal{P}}(G ; x)=F(G ; x)$. 
The characteristic polynomial $\operatorname{char}(G ; x)$ of a graph is the characteristic polynomial of its adjacency matrix and the Laplacian polynomial $\operatorname{Lap}(G ; x)$ is the characteristic polynomial of its Laplace matrix, see [9].

The matching polynomials are defined using $m_{i}(G)$, the number of matchings of $G$ of size $i$.

$$
M(G ; x)=\sum_{i} m_{i}(G) x^{i} \text { and } \mu(G ; x)=\sum_{i}(-1)^{i} m_{i}(G) x^{n-2 i} .
$$

$M(G ; x)$ is the generating matching polynomial and $\mu(G ; x)$ is the matching defect polynomial, see [28].

Let $i n_{i}(G)$ be the number of independent sets of $G$ of size $i$, and $d_{i}(G)$ the number of dominating sets of $G$ of size $i$. We define the independence polynomial IND $(G ; x),[24]$, and the domination polynomial DOM $(G ; x)$, $[3,4,22]$ as

$$
I N D(G ; x)=\sum_{i} i n_{i}(G) x^{i} \text { and } \operatorname{DOM}(G ; x)=\sum_{i} d_{i}(G) x^{i} .
$$

Theorem 2.1. The following are not Harary polynomials of the form $\chi_{\mathcal{P}}(G ; x)$ :

(i) The characteristic polynomial char $(G ; x)$ and the Laplacian polynomial Lap $(G ; x)$.

(ii) The generating matching polynomial $M(G ; x)$ and the defect matching polynomial $\mu(G ; x)$.

(iii) The independence polynomial IND $(G ; x)$.

(iv) The domination polynomial DOM $(G ; x)$.

Proof. We use Proposition 2.1. (i): $\operatorname{char}\left(C_{4} ; x\right)=(x-2) x^{2}(x+2)$ and $\operatorname{Lap}\left(C_{4} ; x\right)=x(x-4)(x-2)^{2}$, hence $\operatorname{char}\left(C_{4} ; 1\right)=\operatorname{Lap}\left(C_{4} ; 1\right)=-3$.

(ii): $M\left(C_{4} ; x\right)=4 x+2 x^{2}$ and $\mu\left(C_{4} ; x\right)=1+4 x+2 x^{2}$,

hence $M\left(C_{4} ; 1\right)=6$ and $\mu\left(C_{4} ; 1\right)=7$,

(iii): $\operatorname{IND}\left(C_{4} ; x\right)=1+4 x+2 x^{2}$, hence $\operatorname{IND}\left(C_{4} ; 1\right)=7$.

(iv): $\operatorname{DOM}\left(K_{2} ; x\right)=2 x+x^{2}$, hence $\operatorname{DOM}\left(K_{2} ; 1\right)=3$.

$D O M$ and $I N D$ are special cases graph polynomials of the form

$$
\mathcal{P}_{\Phi}(G ; x)=\sum_{A \subseteq V(G): \Phi(A)} x^{|A|} .
$$

Graph polynomials of this form are generating functions counting subsets $A \subseteq V(G)$ satisfying a property $\Phi(A)$, in the cases above, that $A$ is an independent, respectively a dominating set, see also [31]. We say that $\Phi$ determines $A$, if for every graph $G$ there is a unique $A \subseteq V(G)$ which satisfies $\Phi(A)$.

Theorem 2.2. Assume that $\Phi$ does not determine $A$, then there is no graph property $\mathcal{P}$ such that for all graphs $G \chi_{\mathcal{P}}(G ; x)=\mathcal{P}_{\Phi}(G ; x)$. Hence $\mathcal{P}_{\Phi}(G ; x)$ cannot be a Harary polynomial.

Proof. By Lemma $2.1 \chi_{\mathcal{P}}(G ; 1) \in\{0,1\}$ for all graphs $G$. However, since $\Phi$ does not determine $A$, there is a graph $H$ with $\mathcal{P}_{\Phi}(H ; 1) \geq 2$.

\section{Are Harary polynomials edge elimination invariants?}

\subsection{Chromatic invariants}

Following [2, Chapter 9.1], a function $f$ which maps graphs into a polynomial ring $\mathcal{R}=\mathcal{K}[\bar{X}]$ with coefficients in a field $\mathcal{K}$ of characteristic 0 is called a chromatic invariant (aka Tutte-Grothendieck invariant) if the following hold.

(i) If $G$ has no edges, $f(G)=1$.

(ii) If $e \in E(G)$ is a bridge, then $f(G)=A \cdot f\left(G_{-e}\right)$.

(iii) If $e \in E(G)$ is a loop, then $f(G)=B \cdot f\left(G_{-e}\right)$.

(iv) There exist $\alpha, \beta \in \mathcal{R}$ such that for every $e \in E(G)$ which is neither a loop nor a bridge we have $f(G)=$ $\alpha \cdot f\left(G_{-e}\right)+\beta \cdot f\left(G_{/ e}\right)$.

(v) Multiplicativity: If $G=G_{1} \sqcup G_{2}$ is the disjoint union of two graphs $G_{1}, G_{2}$ then $f(G)=f\left(G_{1}\right) \cdot f\left(G_{2}\right)$.

Chromatic invariants have a characterization via the Tutte polynomial $T(G ; x, y)$, see $[2$, Chapter 9.1, Theorem 9.5]. 
Theorem 3.1. Let $f$ be a chromatic invariant with $A, B, \alpha, \beta$ indeterminates as above. Then for all graphs $G$

$$
f(G)=\alpha^{|E|-|V|+k(G)} \cdot \beta^{|V|-k(G)} \cdot T\left(G ; \frac{A}{\beta}, \frac{B}{\alpha}\right) .
$$

It follows by a counting argument that not all Harary polynomials are chromatic invariants. We characterize the Harary polynomials which are chromatic invariants in Theorem 3.3 below.

\subsection{Edge elimination invariants}

The Tutte polynomial generalizes the chromatic, flow and other graph polynomials. It is natural to search for polynomials that generalize it, in turn. The Most General Edge Elimination Invariant, introduced in [6], [5] and also known as the $\xi$ polynomial, generalizes the Tutte and the matching polynomials.

Definition 3.1 (Edge Elimination Invariant). Let $F$ be a graph parameter with values in a ring $\mathcal{R}$. $F$ is an EE-invariant if there exist $\alpha, \beta, \gamma \in \mathcal{R}$ such that

$$
F(G)=F\left(G_{-e}\right)+\alpha F\left(G_{/ e}\right)+\beta F\left(G_{\dagger e}\right)
$$

where $e \in E(G)$, with the additional conditions

$$
F(\emptyset)=1, \quad F\left(K_{1}\right)=\gamma, \quad \text { and } \quad F(G \sqcup H)=F(G) \cdot F(H) .
$$

Let $\xi(G ; x, y, z)$ be the graph polynomial

$$
\xi(G ; x, y, z)=\sum_{A, B \subseteq E(G)} x^{c(A \cup B)-\operatorname{cov}(B)} y^{|A|+|B|-\operatorname{cov}(B)} z^{\operatorname{cov}(B)}
$$

where the summation is over $A, B \subseteq E(G)$ such that the vertex subsets $V(A), V(B)$ covered by $A$ and $B$, respectively, are disjoint, $c(A)$ is the number of connected components in $(V(G), A)$, and $\operatorname{cov}(B)$ is the number of covered connected component of $B$, i.e. the number of connected components of $(V(B), B)$.

Theorem 3.2 (See [5]). (i) $\xi(G ; x, y, z)$ is an EE-invariant.

(ii) Every EE-invariant is a substitution instance of $\xi(G ; x, y, z)$ multiplied by some factor $s(G)$ which only depends on the number of vertices, edges and connected components of $G$.

(iii) Both the matching polynomial and the Tutte polynomial are EE-invariants given by

$$
T(G ; x, y)=(x-1)^{-c(E(G))}(y-1)^{-|V(G)|} \xi(G ;(x-1)(y-1), y-1,0),
$$

and

$$
M\left(G ; w_{1}, w_{2}\right)=\xi\left(G ; w_{1}, 0, w_{2}\right)
$$

\subsection{Are Harary polynomials EE-invariants?}

Theorem 3.3. Let $\mathcal{P}$ be a non-trivial (minor closed/monotone/hereditary) graph property. Then $\chi_{\mathcal{P}}$ is an EE-invariant if and only if $\chi_{\mathcal{P}}$ is the chromatic polynomial.

We need a lemma:

Lemma 3.1. Let $\mathcal{P}$ be a non-trivial (minor closed/monotone/hereditary) graph property, and $H$ a forbidden minor, subgraph or induced subgraph of $\mathcal{P}$.

Assume $\mathrm{H}=\mathrm{H}_{1} \sqcup \mathrm{H}_{2}$ with both $\mathrm{H}_{1}$ and $\mathrm{H}_{2}$ in $\mathcal{P}$. Then $\chi_{\mathcal{P}}$ is not multiplicative.

Corollary 3.1. In particular, if $H=E_{n}$ or $H=K_{1} \cup K_{2}$, $\chi_{\mathcal{P}}$ is not multiplicative.

Proof. If $H=H_{1} \sqcup H_{2}$, both $H_{1}, H_{2}$ are minors, subgraphs, and induced subgraphs. Hence we have

$$
0=\chi_{\mathcal{P}}(H, 1)=\chi_{\mathcal{P}}\left(H_{1} \sqcup H_{2}, 1\right)
$$

as $H$ is forbidden, but

$$
\chi_{\mathcal{P}}\left(H_{1}, 1\right) \cdot \chi_{\mathcal{P}}\left(H_{2}, 1\right)=1
$$

Proof of Theorem 3.3. We analyze $H$, a forbidden (minor/subgraph/induced subgraph) of $\mathcal{P}$ with the smallest number of vertices and edges.

The proof distinguishes between cases: 
(i) $H$ is not connected.

(ii) $H=K_{1}$.

(iii) $H=K_{2}$.

(iv) $H=P_{3}$.

(v) $H=K_{3}$.

(vi) $H$ has order $\geq 4$ and size $\geq 1$.

Case (i): $H$ is not connected.

We use Lemma 3.1.

Case (ii): $H=K_{1}$

If $H=K_{1}, \mathcal{P}$ is empty, hence trivial.

Case (iii): $H=K_{2}$

If $H=P_{2}$, then $\chi_{\mathcal{P}}$ is the chromatic polynomial.

Case (iv) $H=P_{3}$

We compute: $\chi_{\mathcal{P}}\left(K_{1}, x\right)=x, \quad \chi_{\mathcal{P}}\left(K_{2}, x\right)=x^{2}$ and

$$
\chi_{\mathcal{P}}\left(P_{3}, x\right)=x^{3}-x, \quad \chi_{\mathcal{P}}\left(K_{1} \cup K_{2}, x\right)=x^{3}
$$

Assuming that $\chi_{\mathcal{P}}$ is an EE-invariant, we can apply the recursive relation to get:

$$
\begin{aligned}
& \chi_{\mathcal{P}}\left(P_{3}, x\right)=\chi_{\mathcal{P}}\left(K_{1} \cup K_{2}, x\right)+\alpha(x) \chi_{\mathcal{P}}\left(K_{2}, x\right)+\beta(x) \chi_{\mathcal{P}}\left(K_{1}, x\right) \\
& =x^{3}+\alpha(x) x^{2}+\beta(x) x \\
& \chi_{\mathcal{P}}\left(K_{1} \cup K_{2}, x\right)=\chi_{\mathcal{P}}\left(E_{3}, x\right)+\alpha(x) \chi_{\mathcal{P}}\left(E_{2}, x\right)+\beta(x) \chi_{\mathcal{P}}\left(K_{1}, x\right) \\
& =x^{3}+\alpha(x) x^{2}+\beta(x) x
\end{aligned}
$$

By combining $(*)$ with $(* *)$ and $(* * *)$

$$
-x=\alpha(x) x^{2}+\beta(x) x=0
$$

which is a contradiction.

Case (v) $H=K_{3}$.

We compute: $\chi_{\mathcal{P}}\left(K_{1}, x\right)=x, \quad \chi_{\mathcal{P}}\left(K_{2}, x\right)=x^{2}$ and

$$
\chi_{\mathcal{P}}\left(K_{3}, x\right)=x^{3}-x, \quad \chi_{\mathcal{P}}\left(K_{1} \cup K_{2}, x\right)=x^{3}
$$

Assuming that $\chi_{\mathcal{P}}$ is an EE-invariant, we can apply the recursive relation to get:

$$
\begin{gathered}
\chi_{\mathcal{P}}\left(K_{3}, x\right)=\chi_{\mathcal{P}}\left(K_{2}, x\right)+\alpha(x) \chi_{\mathcal{P}}\left(K_{2}, x\right)+\beta(x) \chi_{\mathcal{P}}\left(K_{1}, x\right) \\
=x^{3}+\alpha(x) x^{2}+\beta(x) x \\
\chi_{\mathcal{P}}\left(P_{3}, x\right)=\chi_{\mathcal{P}}\left(K_{1} \cup K_{2}, x\right)+\alpha(x) \chi_{\mathcal{P}}\left(K_{2}, x\right)+\beta(x) \chi_{\mathcal{P}}\left(K_{1}, x\right) \\
=x^{3}+\alpha(x) x^{2}+\beta(x) x
\end{gathered}
$$

By combining $(+)$ with $(++)$ and $(+++)$

$$
-x=\alpha(x) x^{2}+\beta(x) x=0
$$

which is a contradiction.

Case (vi): $H$ has order $\geq 4$ and size $\geq 1$.

We note that deleting or contracting or extracting $e$ from $H$, we obtain a graph in $\mathcal{P}$. Hence we can compute:

$$
\begin{gathered}
\chi_{\mathcal{P}}(H, x)=x^{|V(H)|}-x \text { and } \chi_{\mathcal{P}}\left(H_{-e}, x\right)=x^{|V(H)|} \\
\chi_{\mathcal{P}}\left(H_{/ e}, x\right)=x^{|V(H)|-1} \text { and } \chi_{\mathcal{P}}\left(H_{\dagger e}, x\right)=x^{|V(H)|-2}
\end{gathered}
$$

Now we assume that $\chi_{\mathcal{P}}$ is an EE-invariant and get

$$
\begin{gathered}
\chi_{\mathcal{P}}(H, x)=x^{|V(H)|}-x \\
=\chi_{\mathcal{P}}\left(H_{-e}, x\right)+\alpha(x) \cdot \chi_{\mathcal{P}}\left(H_{/ e}, x\right)+\beta(x) \cdot \chi_{\mathcal{P}}\left(H_{\dagger e}, x\right) \\
=x^{V(H)}+\alpha(x) \cdot x^{V(H)-1}+\beta(x) \cdot x^{V(H)-2}
\end{gathered}
$$

for $\alpha(x), \beta(x) \in \mathbb{Z}[x]$ polynomials in $x$.

If $|V(H)| \geq 4$ the coefficient of $x$ in $\left(^{*}\right)$ is -1 ,

and in $(* *)$ it is 0 , which is a contradiction. 
The graph polynomials $C(G ; x)$ and $A(G ; x)$ are Harary polynomials where the property $\mathcal{P}$ contains arbitrarily large cliques.

Proposition 3.1. Both $C(G ; x)$ and $A(G ; x)$ are not multiplicative, hence they are not EE-invariants.

Proof. $C\left(K_{1}, x\right)=A\left(K_{1}, x\right)=x$ and $C\left(K_{1} \sqcup K_{1}, x\right)=A\left(K_{1} \sqcup K_{1}, x\right)=x^{2}-x \neq x^{2}$.

\section{MSOL-definable graph polynomials}

We assume the reader is familiar with second and monadic second-order logic for graphs. A good source is $[12,20,30,31]$. We distinguish between MSOL for the language of graphs, with one binary edge relation $\mathrm{MSOL}_{g}$, and MSOL for the language of hypergraphs $\mathrm{MSOL}_{h}$, with vertices and edges as elements and a binary incidence relation. We also refer to second-order logic $\mathrm{SOL}_{g}, \mathrm{SOL}_{h}$ in a similar way.

A simple univariate $\mathrm{MSOL}_{g}$-definable $\left(\mathrm{MSOL}_{h}, \mathrm{SOL}_{g}, \mathrm{SOL}_{h}\right.$-definable) graph polynomial $F(G ; x)$ is a polynomial of the form

$$
F(G ; x)=\sum_{A \subseteq V(G): \phi(A)} \prod_{v \in I} x,
$$

where $A$ ranges over all subsets of $V(G)$ satisfying $\phi(A)$ and $\phi(A)$ is a $\mathrm{MSOL}_{g}$-formula. $F$ is $\mathrm{MSOL}_{h}$-definable if $A$ ranges over $V(G) \cup E(G)$ and $\phi(A)$ is a $\mathrm{MSOL}_{h}$-formula. $F$ is $\mathrm{SOL}_{g}$-definable if $A$ ranges over $V(G)^{m} . F$ is $\mathrm{SOL}_{h}$-definable if $A$ ranges over $(V(G) \cup E(G))^{m}$.

Examples 4.1. (i) The independence polynomial $I N D(G ; x)=\sum_{i} i n d(G, i) \cdot x^{i}$, can be written as

$$
I N D(G, x)=\sum_{I \subseteq V(G)} \prod_{v \in I} x,
$$

where I ranges over all independent sets of $G$. To be an independent set is $\mathrm{MSOL}_{g}$-definable.

(ii) The matching generating polynomial $M(G ; x)$ is $\mathrm{MSOL}_{h}$-definable, but unlikely to be $\mathrm{MSOL}_{g}$-definable, [32], otherwise it would be fixed parameter tractable for clique-width at most $k$.

For the general case one allows several indeterminates $x_{1}, \ldots, x_{m}$, and gives an inductive definition. One may also allow an ordering of the vertices, but then one requires the definition to be invariant under the ordering, i.e., different orderings still give the same polynomial.

Examples 4.2. The Tutte polynomial is a bivariate $\mathrm{MSOL}_{h}$-definable graph polynomial using an ordering on the vertices, [29]. Similarly, it can be shown that the polynomial $\xi(G ; x, y, z)$ is a trivariate $\mathrm{MSOL}_{h}$-definable graph polynomial using an ordering on the vertices, [5]. For a proof see Theorem 1.3.

A univariate graph polynomial is $\mathrm{MSOL}_{g}$-definable $\left(\mathrm{MSOL}_{h}, \mathrm{SOL}_{g}, \mathrm{SOL}_{h}\right.$-definable) if it is a substitution instance of a multivariate $\mathrm{MSOL}_{g}$-definable $\left(\mathrm{MSOL}_{h}, \mathrm{SOL}_{g}, \mathrm{SOL}_{h}\right.$-definable) graph polynomial.

All we can say about the definability of Harary polynomials is the following:

Proposition 4.1. If $\mathcal{P}$ is $\mathrm{SOL}_{g}$-definable so is the Harary polynomial $\chi_{\mathcal{P}}(G ; x)$.

Proof. We only prove the case where $\mathcal{P}$ is $\mathrm{SOL}_{g}$-definable, the other cases are similar.

Let $\phi$ be the $\mathrm{MSOL}_{g}$-formula which defines $\mathcal{P}$. Let $\Phi(X, E)$ be the formula that says that $X \subseteq V(G)^{2}$ is an equivalence relation on $V(G)$ such that each equivalence class induces a graph satisfying $\phi$. Now we can write

$$
\chi_{\mathcal{P}}(G ; x)=\sum_{X \subseteq V(G)^{2}: \Phi(X, E)} x^{|X|} .
$$

The chromatic polynomial is not $\mathrm{MSOL}_{g}$-definable, In the sequel, we show that many Harary polynomials are not $\mathrm{MSOL}_{g}$-definable.

\section{Connection Matrices}

In this section, we prove for many Harary polynomials that they are not $\mathrm{MSOL}_{q}$-polynomials.

We use tools from [30]. Let $G_{i}$ be an enumeration of all finite graphs (up to isomorphisms). We denote by $G_{i} \sqcup G_{j}$ the disjoint union, and by $G_{i} \bowtie G_{j}$ the join of $G_{i}$ and $G_{j}$. 
Let $F=F(G ; x) \in \mathbb{Z}[x]$ be a graph polynomial. Let $\mathcal{H}(\bowtie, F)$ be the infinite matrix where rows and columns are labeled by $G_{i}$. Then we define

$$
\begin{aligned}
\mathcal{H}(\bowtie, F)\left(G_{i}, G_{j}\right) & =F\left(G_{i} \bowtie G_{j} ; x\right), \\
\mathcal{H}(\sqcup, F)\left(G_{i}, G_{j}\right) & =F\left(G_{i} \sqcup G_{j} ; x\right) .
\end{aligned}
$$

$\mathcal{H}(\bowtie, F)$, respectively $\mathcal{H}(\sqcup, F)$, is called a connection matrix also known as Hankel matrix.

Theorem 5.1 (See [30]). If $F(G ; x)$ is $\mathrm{MSOL}_{g}$-definable, then $\mathcal{H}(\bowtie, F)$ and $\mathcal{H}(\sqcup, F)$ have finite rank over the ring $\mathbb{Z}[x]$.

The following lemmas are needed for Theorem 5.2.

Lemma 5.1 (See [30, Lemma 9.4]). Given a graph polynomial $F$, and an infinite sequence of non-isomorphic graphs $H_{i}, i \in \mathbb{N}$, let $f: \mathbb{N} \rightarrow \mathbb{N}$ be an unbounded function such that for every $k \in \mathbb{N}, F\left(H_{i} \bowtie H_{j}, k\right)=0$ if and only if $i+j>f(k)$.

Then the matrix $\mathcal{H}(\bowtie, p)$ has infinite rank.

The same also holds when $\bowtie$ is replaced by the disjoint union $\sqcup$.

Given a graph $H$ we denote by $F o r b^{s u b}(H)\left(F o r b^{i n d}(H)\right)$ the class of graphs which do not contain an (induced) subgraph isomorphic to $H$. If $H$ is a complete graph the two classes coincide, and we omit the superscript.

We now prove specific cases where we can apply Lemma 5.1 with $H_{i}=K_{i}$ the complete graph on $i$ vertices.

Lemma 5.2. (i) Let $\mathcal{P}_{1} \subseteq \operatorname{Forb}\left(K_{h}\right)$. Then $\chi_{\mathcal{P}_{1}}\left(K_{i} ; k\right)=0$ if and only if $i>h k$.

(ii) Let $\mathcal{P}_{2} \subseteq$ Forb $^{\text {sub }}(H)$ for some connected graph $H$ on $h$ vertices. Then $\chi_{\mathcal{P}_{2}}\left(K_{i} ; k\right)=0$ if and only if $i>h k$.

Proof. If we partition a set of size $i>h k$ into $k$ disjoint sets, at least on of these sets has size $>h$. Hence, if we partition $K_{i}$, at least one of these sets induces a $K_{h}$. So hence $\chi_{\mathcal{P}_{1}}\left(K_{i} ; k\right)=0$. Since $H$ is a subgraph of $K_{h}$, $\chi_{\mathcal{P}_{2}}\left(K_{i} ; k\right)=0$.

Note that for $\mathcal{P}=\operatorname{Forb}\left(K_{2}\right)$ this is the chromatic polynomial.

Theorem 5.2. Let $\mathcal{P}$ be a non-trivial graph property. If $\mathcal{P}$ is (i) monotone, (ii) ultimately clique-free, or (iii) weakly sparse, the Harary polynomial $\chi_{\mathcal{P}}(G ; x)$ is not $\mathrm{MSOL}_{g}$-definable.

Proof. (i): If $P$ is non-trivial and monotone there is a connected graph $H$ with $P \subset \operatorname{Forb}^{s u b}(H)$. By Lemma 5.2 (ii) we get $\chi_{P}\left(K_{i} ; k\right)=0$ if and only if $i>h k$.

By Lemma 5.1, $\mathcal{H}\left(\bowtie, \chi_{P}(G ; x)\right)$ has infinite rank. Now we use Theorem 5.1.

(ii): $\mathcal{P} \subseteq \operatorname{Forb}\left(K_{h}\right)$, hence by Lemma $5.2(\mathrm{i})$, we get again $\chi_{P}\left(K_{i} ; k\right)=0$ if and only if $i>h k$. Then we proceed as in (i).

(iii): $\mathcal{P}$ is ultimately clique-free hence there is $h \in \mathbb{N}$ with $P \subseteq F \operatorname{Forb}\left(K_{h}\right)$. So we proceed as in (ii).

The graph polynomials $C(G ; x)$ and $A(G ; x)$ are Harary polynomials where the property $\mathcal{P}$ contains graphs of maximal density.

Proposition 5.1. Both $C(G ; x)$ and $A(G ; x)$ are not $\mathrm{MSOL}_{g}$-definable.

Proof. In both cases we look at the graph $M_{n}$ of order $2 n$ which consists of $n$ disjoint copies of $K_{2}$. We note that $M_{i} \sqcup M_{j}=M_{i+j}$, and we get $C\left(M_{n} ; k\right)=A\left(M_{n} ; k\right)=0$ for $n>2 k$. So we can apply Lemma 5.1 with the join replaced by the disjoint union.

\section{Conclusions and Open Problems}

We have initiated a systematic study of Harary polynomials.

In this paper, we have shown that among the Harary polynomials $\chi_{\mathcal{P}}$ with $\mathcal{P}$ hereditary monotone, or minor-closed, the chromatic polynomial is the only EE-invariant.

We have also shown that the Harary polynomials $\chi_{\mathcal{P}}$ are not $\mathrm{MSOL}_{g}$-definable if $\mathcal{P}$ is either monotone, ultimately clique-free, or weakly sparse. This includes the chromatic polynomial. However, the chromatic polynomial is $\mathrm{MSOL}_{h}$-definable.

Question 6.1. Is there a Harary polynomial, different from the chromatic polynomial, which is $\mathrm{MSOL}_{h}$ definable and/or an EE-invariant?

We suspect (but do not conjecture) that the chromatic polynomial is the only Harary polynomial which is an EE-invariant?

In future research, we continue the study of the complexity of evaluating Harary polynomials, initiated in $[18]$. 


\section{Acknowledgments}

We would like to thank P. Tittmann for his careful reading of drafts of this paper. We would also like to thank three anonymous referees of the previous version of this paper for their valuable and justified comments. This research profited also a lot from two Dagstuhl Seminars:

- Dagstuhl Seminar 16241, June 12-17, 2016,

Graph Polynomials: Towards a Comparative Theory

- Dagstuhl Seminar 19401, September 29-October 4, 2019, Comparative Theory for Graph Polynomials

\section{References}

[1] D. Achlioptas, The complexity of G-free colourability, Discrete Math. 165 (1997), 21-30.

[2] M. Aigner, A course in enumeration, Graduate Texts in Mathematics, Springer, 2007.

[3] S. Alikhani, Dominating sets and domination polynomials of graphs, Universiti Putra Malaysia, 2009.

[4] J. Arocha and B. Llano, Mean value for the matching and dominating polynomial, Discuss. Math. Graph Theory 20:1 (2000), 57-69.

[5] I. Averbouch, B. Godlin and J.A. Makowsky, A most general edge elimination polynomial, International Workshop on Graph-Theoretic Concepts in Computer Science, Springer, 2008, 31-42.

[6] I. Averbouch, B. Godlin and J.A. Makowsky, An extension of the bivariate chromatic polynomial, European J. Combin. 31:1 (2010), 1-17.

[7] I. Averbouch, T. Kotek, J.A. Makowsky and E. Ravve, The universal edge elimination polynomial and the dichromatic polynomial, Electron. Notes Discrete Math. 38 (2011), 77-82.

[8] F. Bencs, One more remark on the adjoint polynomial, European J. Combin. 65 (2017), 253-258.

[9] A.E. Brouwer and W.H. Haemers, Spectra of graphs, Springer Science \& Business Media, 2011.

[10] J.I. Brown, The complexity of generalized graph colorings, Discrete Appl. Math. 69:3 (1996), 257-270.

[11] J.I. Brown and D.G. Corneil, On generalized graph colorings, J. Graph Theory 11:1 (1987), 87-99.

[12] B. Courcelle and J. Engelfriet, Graph structure and monadic second-order logic: a language-theoretic approach 138, Cambridge University Press, 2012.

[13] B. Courcelle, J.A. Makowsky and U. Rotics, On the fixed parameter complexity of graph enumeration problems definable in monadic second-order logic, Discrete Appl. Math. 108:1-2 (2001), 23-52.

[14] R.G. Downey and M.R. Fellows, Fundamentals of Parameterized Complexity, Springer Science \& Business Media, 2013.

[15] A. Farrugia, Vertex-partitioning into xed additive induced-hereditary properties is NP-hard, Electron. J. Combin. 11 (2004), \#19.

[16] J. Flum and M. Grohe, Parameterized complexity theory, Springer Science \& Business Media, 2006.

[17] A. Frieze and M. Karoński, Introduction to random graphs, Cambridge University Press, 2016.

[18] A. Goodall, M. Hermann, T. Kotek, J.A. Makowsky and S.D. Noble, On the complexity of generalized chromatic polynomials, Adv. in Appl. Math. 94 (2018), 71-102.

[19] F. Harary, Conditional Colorability in Graphs: Graphs and Application, Proceedings of First Colorado Symposium of Graph Theory, New York: John Wiley \& Sons Inc, 1985.

[20] T. Kotek, Logic-Based Computation of Graph Polynomials, In Yogtang Shi, Matthias Dehmer, Xueliang Li, and Ivan Gutman, Graph Polynomials, 151-176, CRC Press, 2016.

[21] T. Kotek, J.A. Makowsky and B. Zilber, On counting generalized colorings, In M. Grohe and J.A. Makowsky, Model theoretic methods in finite combinatorics, volume 558 in Contemporary Mathematics, 207-242, American Mathematical Society, 2011.

[22] T. Kotek, J. Preen, F. Simon, P. Tittmann and M. Trinks, Recurrence Relations and Splitting Formulas for the Domination Polynomial, Electron. J. Combin. 19:3 (2012), P47.

[23] T. Kovári, V. Sós and P. Turán, On a problem of K. Zarankiewicz, Colloq. Math. 3 (1954), 50-57.

[24] V.E. Levit and E. Mandrescu, The independence polynomial of a graph-a survey, Proceedings of the 1st International Conference on Algebraic Informatics, volume 233254, 231-252, Aristotle Univ. Thessaloniki Thessaloniki, 2005.

[25] N. Linial, Hard enumeration problems in geometry and combinatorics, SIAM Journal on Algebraic Discrete Methods 7:2 (1986), 331-335. 
[26] N. Linial, J. Matoušek, O. Sheffet and G. Tardos, Graph colouring with no large monochromatic components, Combin. Probab. Comput. 17:4 (2008), 577-589.

[27] R.-Y. Liu and L.-C. Zhao, A new method for proving chromatic uniqueness of graphs, Discrete Math. 171:1-3 (1997), 169-177.

[28] L. Lovász and M.D. Plummer, Matching theory, volume 367, American Mathematical Soc., 2009.

[29] J.A. Makowsky, Coloured Tutte polynomials and Kauffman brackets for graphs of bounded tree width, Discrete Appl. Math. 145:2 (2005), 276-290.

[30] J.A. Makowsky and T. Kotek, Connection Matrices and the Definability of Graph Parameters, Log. Methods Comput. Sci. 10, 2014.

[31] J.A. Makowsky, E.V. Ravve and T. Kotek, A logician's view of graph polynomials, Ann. Pure Appl. Logic 170:9 (2019), 1030-1069.

[32] J.A. Makowsky, U. Rotics, I. Averbouch and B. Godlin, Computing graph polynomials on graphs of bounded clique-width, International Workshop on Graph-Theoretic Concepts in Computer Science, 191204, Springer, 2006.

[33] J.A. Makowsky and B. Zilber, Polynomial invariants of graphs and totally categorical theories, MODNET Preprints 21, 2006.

[34] J. Nešetřil and P.O. De Mendez, Sparsity: graphs, structures, and algorithms, volume 28, Springer Science \& Business Media, 2012.

[35] J. Nešetřil and P.O. De Mendez, Structural sparsity, Russian Math. Surveys 71:1 (2016), 79.

[36] J. Nešetřil, P.O. De Mendez, R. Rabinovich and S. Siebertz, Classes of graphs with low complexity: the case of classes with bounded linear rankwidth, European J. Combin. 91 (2021), 103223.

[37] G.C. Rota, On the foundations of combinatorial theory I. Theory of Möbius functions, Zeitschrift für Wahrscheinlichkeitstheorie und verwandte Gebiete 2:4 (1964), 340-368.

[38] F. Simon, P. Tittmann and M. Trinks, Counting Connected Set Partitions of Graphs, Electron. J. Combin., P14-P14, 2011.

[39] J.A. Telle and Y. Villanger, FPT algorithms for domination in sparse graphs and beyond, Theoret. Comput. Sci. 770 (2019), 62-68.

[40] M. Trinks, The Covered Components Polynomial: A New Representation of the Edge Elimination Polynomial, Electron. J. Combin., P50-P50, 2012.

[41] M. Trinks, Proving properties of the edge elimination polynomial using equivalent graph polynomials, arXiv preprint arXiv:1205.2205, 2012.

[42] P. Turán, On an extremal problem in graph theory, Matematikai és Fizikai Lapok 48 (1941), 436-452.

\section{A. Proof of Theorem 1.3}

Theorem 1.3 states that the most general EE-invariant $\xi(G ; x, y, z)$ is $\mathrm{MSOL}_{h}$-definable for graphs with a linear order on the vertices. Furthermore, this definition is invariant under the particular order of the vertices.

Proof.

$$
\begin{aligned}
\xi(G ; x, y, z)= & \sum_{A, B \subseteq E(G)} x^{c(A \cup B)-c_{\operatorname{cov}}(B)} y^{|A|+|B|-c_{\operatorname{cov}}(B)} z^{c_{\operatorname{cov}}(B)}= \\
& \sum_{A, B \subseteq E(G)} x^{c(A \cup B)} y^{|A|+|B|}\left(\frac{z}{x y}\right)^{c_{\text {cov }}(B)}
\end{aligned}
$$

Let $(V(G), E(G), \operatorname{Ord}(G))$ be a graph with a linear ordering of the vertices.

- Let $\phi(A, B)$ be the formula $\operatorname{cov}(A) \cap \operatorname{cov}(B)=\emptyset$ with $\operatorname{cov}(A, v)=\exists v_{1}, v_{2}\left(\left(v_{1}, v_{2}\right) \in A \wedge\left(v=v_{1} \vee v=v_{2}\right)\right)$ where $A$ ranges over sets of edges, and $v, v_{1}, v_{2}$ range over vertices.

- We write for sets of edges $A, B$ :

$$
X^{|A|}=\prod_{e: e \in A} X \text { and } X^{|A|+|B|}=\prod_{e: e \in A \sqcup B} X
$$

where $A \sqcup B$ is the disjoint union of $A$ and $B$. 
- We write for a vertex $v$ and a set of edges $A$ :

$$
X^{c(F)}=\prod_{v: \phi_{c}(F, v)} X
$$

where $\phi_{c}(F, v)$ says that $v$ is the first vertex of a connected component of the graph $(V(G), F)$. If $F=A \sqcup B$ we use instead $\psi_{c}(A, B, v)$ which says $\exists F\left(\phi_{c}(F, v) \wedge F=A \sqcup B\right)$.

- We write for a vertex $w$ and a set of edges $B$ :

$$
X^{\operatorname{cov}(A)}=\prod_{w: \phi_{\operatorname{cov}}(B, w)} X
$$

where $\phi_{c o v}(B, w)$ says that $w$ is the first vertex of a connected component of the graph $(V(B), B)$.

Then we can write

$$
\xi(G ; x, y, z)=\sum_{A, B: \phi(A, B)}\left(\prod_{u: \phi_{c}(A, B, u)} x \cdot \prod_{e: e \in A \sqcup B} y \cdot \prod_{w: \phi_{c o v}(B, w)} \frac{z}{x y}\right)
$$

